\title{
Sistema de iluminación para obtención de imágenes optoacústicas basado en LEDs y EDU-CIAA
}

\author{
Illumination system for optoacoustic imaging based on LEDs and EDU-CIAA \\ Emiliano Gasparovic*1, Roberto M. Insabella*, Martín G. González*† \\ *Universidad de Buenos Aires, Facultad de Ingeniería, \\ Grupo de Láser, Óptica de Materiales y Aplicaciones Electromagnéticas (GLOmAe) \\ Paseo Colón 850, C1063ACV, Buenos Aires, Argentina \\ ${ }^{\dagger}$ Consejo Nacional de Investigaciones Científicas y Técnicas, (CONICET) \\ Godoy Cruz 2290, C1425FQB, Buenos Aires, Argentina \\ 1 egasparovicefi.uba.ar \\ Recibido: 14/10/20; Aceptado: 28/10/20
}

\begin{abstract}
Optoacoustic imaging is a hybrid technique that benefits from both the versatile optical contrast and the high spatial resolution associated with the low propagation dispersion of ultrasonic waves. Usually, high energy lasers $(>10 \mathrm{~mJ})$ are used as a light source which are expensive, non-portable and have a low repetition rate $(\sim 20 \mathrm{~Hz})$. In recent years, the use of light-emitting diodes (LEDs) as substitute has been studied because of they are less expensive, more reliable and compact, and they can provide thousands of pulses in a second for real-time imaging. This paper presents the implementation of an illumination system based on LEDs and the embedded system EDU-CIAA. The developed system is capable of emitting short light pulses $(<1 \mu$ s) with great intensity and high frequency $(>1 \mathrm{kHz})$.
\end{abstract}

Keywords: optoacoustic; LED; Embedded System.

Resumen- La obtención de imágenes optoacústicas es una técnica híbrida que se beneficia tanto del rico y versátil contraste óptico como de la alta resolución espacial asociada a la baja dispersión de propagación de las ondas ultrasónicas. Usualmente, se utilizan láseres de elevada energía $(>10$ mJ) como fuente de luz los cuales son caros, no portátiles y tienen una baja tasa de repetición $(\sim 20 \mathrm{~Hz})$. En los últimos años se ha estado investigando el uso de diodos emisores de luz (LEDs) como sustituto ya que son menos costosos, más confiables y compactos, $y$ pueden proporcionar miles de pulsos en un segundo para obtener imágenes en tiempo real. En esta trabajo se presenta la implementación de un sistema de iluminación basado en LEDs y en el sistema embebido EDU-CIAA que es capaz de emitir pulsos de luz de corta duración $(<1 \mu \mathbf{s})$, con gran intensidad y elevada frecuencia ( $>1 \mathrm{kHz})$.

Palabras clave: optoacústica; LED; sistema embebido.

\section{INTRODUCCIÓN}

La obtención de imágenes optoacústicas $(\mathrm{OA})$ es un enfoque diferente y muy prometedor que proporciona mapas de absorción óptica de alta resolución mediante la detección de ondas de ultrasonido resultantes de la expansión térmica producida por la irradiación de muestras con pulsos cortos de luz [1]. Ejemplos de campos de aplicación son la tomografía y la microscopía [2] donde es posible alcanzar resoluciones de algunas decenas de micrómetros [3], [4]. Dado que el método se basa en la detección de ondas acústicas, cuya longitud de dispersión en muestras biológicas es de 2 a 3 órdenes de magnitud menor que las luminosas, las imágenes OA tienen una alta resolución preservando el alto contraste de absorción óptica [1].

Todo sistema OA se encuentra formado por tres partes esenciales: i) una fuente de excitación lumínica, ii) un sistema de detección para la captura de las señales ultrasónicas y iii) un sistema de procesamiento de los datos para la obtención de la imagen. Como iluminación se suelen usar láseres pulsados de Nd:YAG con generador de segunda armónica $(532 \mathrm{~nm})$ y con pulsos cortos $(\sim 5 \mathrm{~ns})$ y frecuencias del orden de $10 \mathrm{~Hz}$.

La duración del pulso láser para la obtención de imágenes OA debe ser menor que los tiempos característicos de confinamiento térmico y elástico [1], lo que implica un láser que emita en el espectro visible (VIS) y en el infrarrojo cercano (NIR) con una duración de pulso menor al microsegundo. Los láseres de excitación tradicionales de alta energía utilizados en sistemas OA son caros, no portátiles y tienen una baja tasa de repetición $\left(\begin{array}{llll}10 & -100 \mathrm{~Hz}\end{array}\right)$ [5]. La limitada frecuencia es un problema cuando se desean imágenes dinámicas de los tejidos, como por ejemplo el latido del corazón [6]. Los láseres Nd:YAG de 1064 nm o $532 \mathrm{~nm}$ ofrecen una opción económica, pero al emitir en una longitud de onda fija, se limita su aplicación principalmente a la obtención de imágenes anatómicas. Por otro lado, para tener una mayor profundidad de penetración de la luz en el tejido es necesario contar con una fuente de luz que emita en la ventana espectral entre $500 \mathrm{~nm}$ y $900 \mathrm{~nm}$. Esto requiere combinar el láser Nd:YAG con un oscilador óptico paramétrico (OPO) [7] que encarece drásticamente el sistema.

En los últimos años se ha estado investigando fuentes de luz basadas en diodos emisores de luz (LEDs) como sustituto del láser convencional Nd:YAG/OPO [5], [8]-[10]. Los LEDs ofrecen una reducción significativa en el costo, tienen frecuencias de pulso más altas $(>1 \mathrm{kHz})$, son más estables que las fuentes de luz basadas en OPO y permiten sistemas portátiles y de múltiples longitudes de onda [5]. 


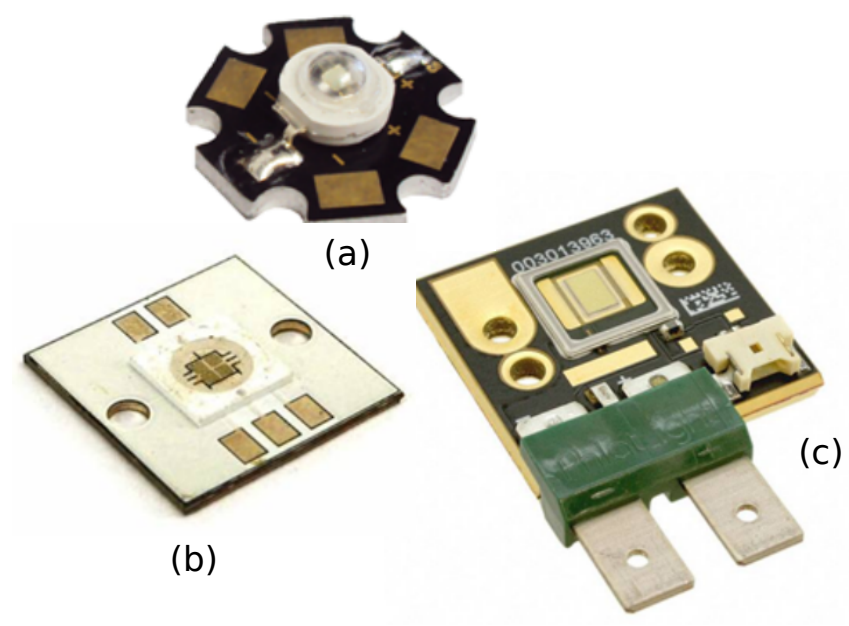

Fig. 1. Imágenes de los LEDs probados en este trabajo. (a) y (b) LEDs genéricos de $3 \mathrm{~W}$ y $5 \mathrm{~W}$, respectivamente. (c) LED Luminus modelo CBT120-G-C11. Todos emiten en una longitud de onda de $\sim 525 \mathrm{~nm}$.

Además, ya se han conseguido con éxito demostraciones del potencial de estas fuentes de luz, como por ejemplo para identificar la artritis inflamatoria en las articulaciones de la mano humana [11].

En este trabajo se presenta el desarrollo de un sistema de excitación basado en LEDs apto para ser usado en la obtención de imágenes OA. Se diseñó e implementó un sistema electrónico de excitación y control de los LEDs para conseguir pulsos a frecuencias de hasta $10 \mathrm{kHz}$ y de duración menor al microsegundo. Para la etapa de control se utilizó el sistema embebido EDU-CIAA [12]. Para verificar el funcionamiento del dispositivo, se realizó la medición de una señal OA proveniente de un fantoma con características ópticas y acústicas similares a las encontradas en muestras biológicas.

\section{MATERIALES Y MÉTOdos}

Para la elección de los LEDs se tuvo en cuenta la mínima corriente pico para conseguir una amplitud de señal OA que pueda ser medida por los sistemas de detección implementados en trabajos previos [13]-[16]. Esto depende principalmente de la potencia de luz de los dispositivos comerciales para las longitudes de onda de emisión de interés (500 nm - $900 \mathrm{~nm})$. Otro criterio para la elección fue que la inductancia parásita del dispositivo fuera lo suficientemente baja como para permitir el paso de una elevada corriente pulsada de corta duración $(<1 \mu \mathrm{s})$. En todos los casos se utilizaron LEDs que emitan con una longitud de onda correspondiente al color verde, ya que generalmente poseen mayor flujo luminoso a similares valores de corriente en comparación con otras longitudes de onda en el espectro visible y en el infrarrojo. Se comenzó estudiando LEDs genéricos con potencias de $3 \mathrm{~W}$ y $5 \mathrm{~W}$ que se consiguen en el mercado local (imágenes (a) y (b) de la Fig. 1). Dado que los LEDs genéricos carecen de hojas de datos, se realizaron ensayos para ver su performance. En el caso del LED de $3 \mathrm{~W}$, trae incluido un disipador (carcasa negra) que en las pruebas limitaba la corriente máxima alcanzable debido a la resistencia e inductancia parásita que introducía en el circuito. A fin de confirmar esta hipótesis se separó

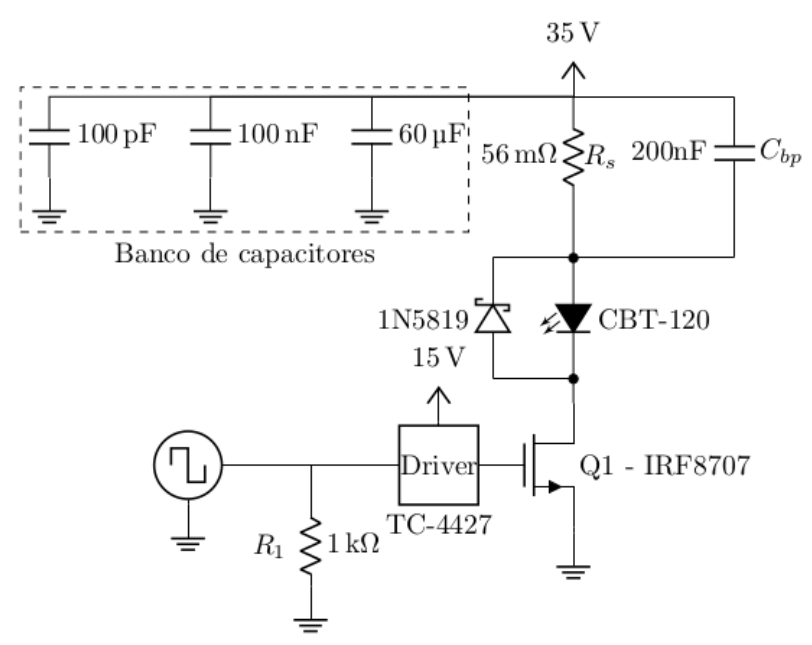

Fig. 2. Diagrama del circuito para la obtener pulsos de corriente para la conmutación del LED.

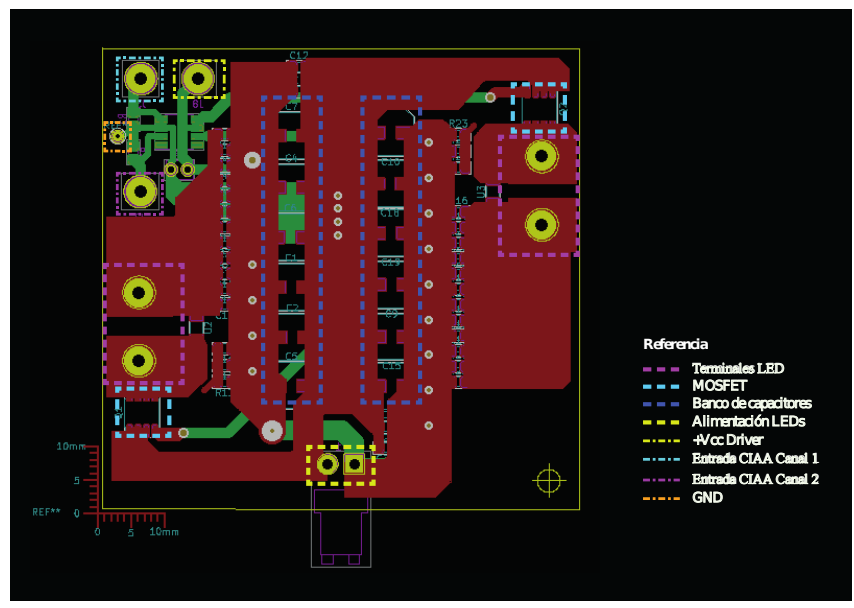

Fig. 3. Layout del prototipo del circuito de excitación para dos LEDs Luminus modelo CBT-120-G-C11.

el encapsulado del LED del disipador. Si bien con ello se incrementó el valor de corriente máxima obtenida, no resultó suficiente para la aplicación deseada. Esto posiblemente se deba a que el encapsulado aún incluye largos terminales que se encuentran conectados por finos hilos conductores. Una situación similar se encontró en el LED genérico de $5 \mathrm{~W}$.

Finalmente, descartando los LEDs del mercado local, se probó el LED Luminus modelo CBT-120-G-C11 [17], encontrándose que posee una potencia de emisión adecuada y una inductancia parásita despreciable. Este LED posee un área de $12 \mathrm{~mm}^{2}$ e irradia un flujo luminoso continuo máximo de $3640 \mathrm{~lm}$ a una longitud de $(525 \pm 17) \mathrm{nm}$ (imagen (c) de la Fig. 1). Asimismo, se aprovechó el requerimiento de la corta duración, necesario en sistemas OA (bajo ciclo de trabajo), para obtener potencias instantáneas mayores a las especificadas por el fabricante [17].

Para la obtención de pulsos de corriente para la conmutación del LED, se diseñó e implementó el circuito que se muestra en la Fig. 2. Dado que estos pulsos implican un gran suministro de corriente instantánea, se tuvo especial cuidado en reducir lo más posible las resistencias y las inductancias parásitas de la placa. En este sentido, se confeccionaron pistas de gran ancho, y se usaron componentes de montaje 


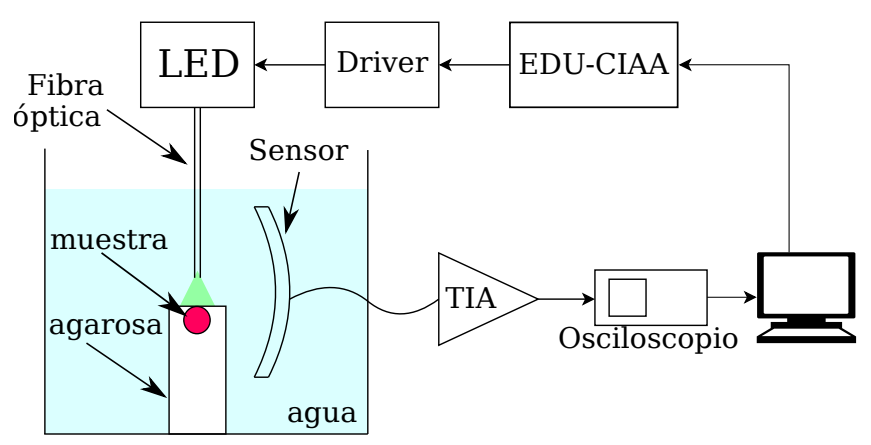

Fig. 4. Esquema experimental para medición de señales OA generadas con el sistema de iluminación implementado en este trabajo.

superficial (ver layout presentado en la Fig. 3). Para la conmutación del encendido y apagado del LED se optó por el transistor MOSFET IRF8707 $\left(Q_{1}\right)$ [18] que posee características deseables para los tipos de pulsos que se desean generar. Asimismo, para conmutar rápidamente $Q_{1}$, se usó el driver TC4427 [19].

El sistema de iluminación requerido debe poseer una señal de referencia consistente en pulsos de duración menor al microsegundo, con la posibilidad de variar este valor manteniendo una resolución aceptable. Esto fue realizado aprovechando las capacidades del sistema embebido EDUCIAA, que permite generar con facilidad una señal del tipo deseada, con una resolución aproximada de $5 \mathrm{~ns}$. A modo comparativo, esto no sería posible de implementar con idénticos resultados en un Arduino dado que sus microcontroladores poseen una menor frecuencia de trabajo. Otra ventaja del sistema EDU-CIAA, es la disponibilidad de salidas con modulación por ancho de pulsos (PWM), que sirve para expandir las prestaciones del sistema de iluminación a un arreglo con distintas frecuencias y anchos de pulso.

El sistema fue probado a través de la medición de señales OA usando el arreglo experimental presentado en la Fig. 4. Para la detección de ultrasonido se utilizó un sensor piezoeléctrico enfocado (foco $=23 \mathrm{~mm}$ ) con características similares al descripto en [20]. El sensor piezoeléctrico y la muestra son sumergidos en un recipiente que contiene agua destilada. El fantoma es una esfera de colorante rojo ( $\sim 1.25 \mathrm{~mm}$ de diámetro) embebida en un bloque de agarosa, siguiendo el procedimiento detallado en [21]. De esta manera, la muestra posee propiedades ópticas y acústicas similares a las encontradas en tejido biológico [22]. Para otorgar mayor flexibilidad en la iluminación, se usó una fibra óptica de plástico de $5 \mathrm{~mm}$ de diámetro para irradiar la muestra. La energía del pulso de luz se midió con un detector piroeléctrico (Coherent J-10MB-LE) [23] y para la caracterización del ancho de pulso se utilizó un fotodiodo rápido (Newport 818-BB-21) [24]. Una base de traslación $\mathrm{XYZ}$ permite ajustar la posición del sistema de detección compuesto por el sensor piezoeléctrico y un amplificador de transimpedancia (TIA FEMTO HCA-100MHz-50K-C) [25]. La señal de salida es digitalizada por un osciloscopio (Tektronix TDS 2024, 2 GS/s, $200 \mathrm{MHz}$ ) y procesada en una computadora. Finalmente, la velocidad del sonido en el agua se determinó midiendo su temperatura con una termocupla calibrada. Para todas las mediciones llevadas a cabo en este

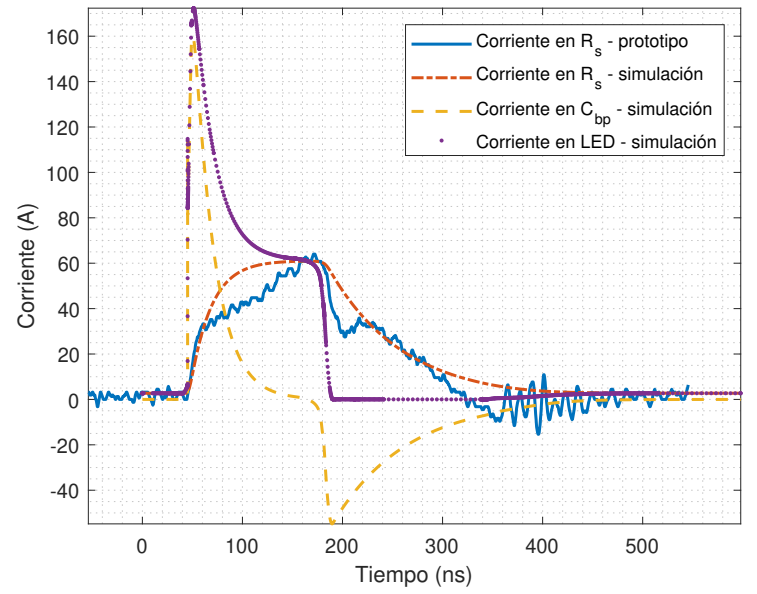

Fig. 5. Corrientes medidas y simuladas para un ancho de pulso de referencia generado por al EDU-CIAA de $94 \mathrm{~ns}$.

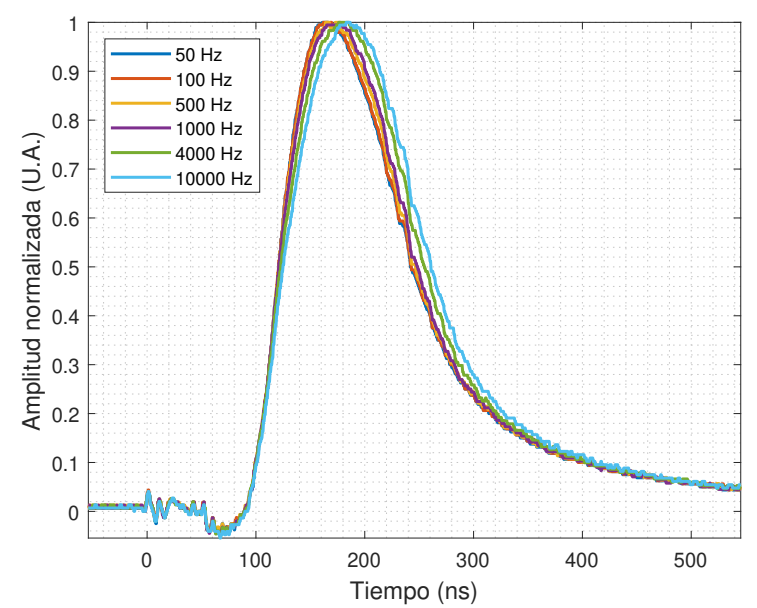

Fig. 6. Medición de la intensidad de luz emitida por el LED. La duración del pulso de referencia generado por el sistema EDU-CIAA fue de 44 ns.

trabajo el valor de la temperatura fue de $24^{\circ} \mathrm{C}$, lo que da una velocidad del sonido de $1494 \mathrm{~m} / \mathrm{s}$ [26].

\section{RESUltados}

Para caracterizar el sistema de iluminación implementado se llevaron a cabo un conjunto de mediciones. En primer lugar, se realizaron simulaciones y mediciones eléctricas sobre el circuito mostrado en la sección II. La generación de pulsos ópticos cortos con un LED está limitada por sus tiempos intrínsecos de encendido (subida) y apagado (bajada). Esta limitación se debe principalmente al tiempo de recombinación de los portadores y a la constante de tiempo RC del circuito equivalente del LED [27]. En este sentido, para reducir el tiempo de subida de la señal óptica (encendido del LED), se usó el método de sobreimpulso al inicio del pulso de corriente. Esta técnica se realiza agregando una capacitancia $\left(C_{b p}\right)$ en paralelo a la resistencia limitadora de corriente $R_{s}$ (ver Fig. 2). En la Fig. 5 se presenta la medición y simulación de las corrientes a través de la resistencia limitadora $R_{s}$, de $C_{b p}$ y del LED para un ancho de pulso de referencia generado por la EDU-CIAA de $94 \mathrm{~ns}$ y una frecuencia de $1 \mathrm{kHz}$. Para la realización 


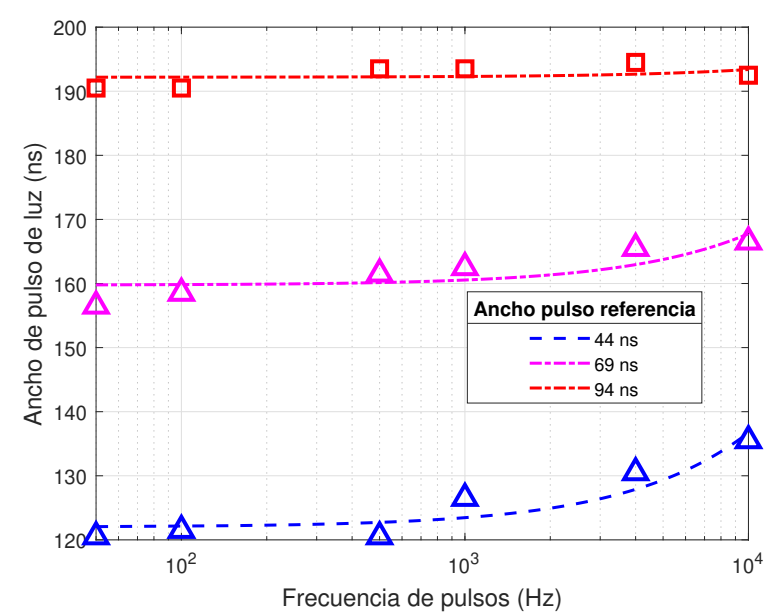

Fig. 7. Variación del ancho del pulso de luz vs. la frecuencia.

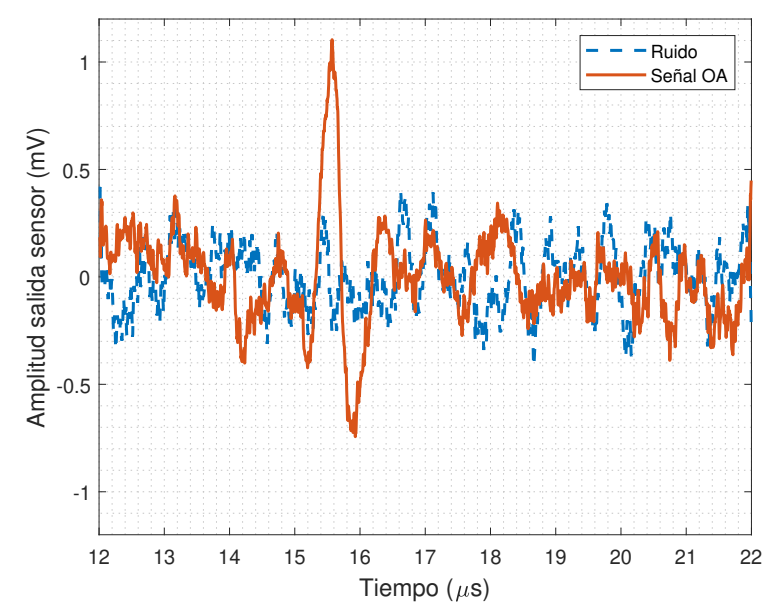

Fig. 8. Señal OA captada por el sistema de detección para un ancho de pulso de luz de $200 \mathrm{~ns}$, una frecuencia de $1 \mathrm{kHz}$ y $1 \mu \mathrm{J}$ de energía y promediadas 5000 veces.

de las simulaciones se utilizó el programa LTspice [28]. Las simulaciones de las corrientes en $C_{b p}$ y en el LED son un ejemplo de como funciona el método del sobreimpulso. Asimismo, para relevar la corriente media entregada al LED, se midió la caída de tensión en la resistencia $R_{s}$. En la Fig. 5 se puede observar que la curva simulada coincide muy bien con la medición. Por otro lado, el ensachamiento del pulso de corriente respecto del valor de referencia, se debe principalmente al driver utilizado que también introduce un retardo de $\sim 50 \mathrm{~ns}$.

En segundo lugar, se analizó la duración del pulso de luz en función de la frecuencia. En la Fig. 6 se muestran las mediciones de la intensidad de luz emitida por el LED para distintos valores de frecuencia donde es posible observar una variación del ancho del pulso óptico. Como los pulsos no son cuadrados, se relevó el ancho a la mitad de su valor máximo (FWHM). En la Fig. 7 se presentan los resultados obtenidos para tres anchos de pulso de referencia generado por el sistema EDU-CIAA (44 ns, 69 ns y 94 ns). Se puede apreciar que se produce un ensanchamiento del pulso de luz respecto del valor de referencia y que su efecto se hace más notorio a frecuencias $>1 \mathrm{kHz}$. Como se puede ver en la Fig. 6, este efecto se debe principalmente al aumento del tiempo de apagado del LED. Para mejorar el tiempo de caída óptica es común cortocircuitar el LED durante la fase de apagado [29]. Sin embargo, cuando se utilizan LEDs de multi-pozos cuánticos (MQW) de alta intensidad y con corrientes máximas superiores a 1 A (como el LED CBT120-G-C11), esta estrategia no tiene el resultado deseado [30]. Otro enfoque es agregar un inductor y una resistencia entre el cátodo y la fuente de forma de descargar el LED con una corriente inversa durante la fase de apagado [30] pero, en este trabajo, fue probada sin éxito.

También se midió la energía emitida por el conjunto LED + fibra óptica y se observó la misma dependencia con la frecuencia, o sea, se tiene un valor aproximadamente constante hasta $1 \mathrm{kHz}$ (por ejemplo, para 200 ns se midió 1 $\mu \mathrm{J} \pm 0.2 \mu \mathrm{J})$ y para frecuencias mayores, la energía medida posee una tendencia similar a la mostrada en la Fig. 7.

En último lugar, se probó el sistema de iluminación implementado en el arreglo experimental presentado en la Fig. 4 y descripto en la sección II. En la Fig. 8 se muestra la señal OA medida para un ancho de pulso de luz de 200 ns, una frecuencia de $1 \mathrm{kHz}$ y $1 \mu \mathrm{J}$ de energía. En la misma figura también se ha incluido la respuesta del sistema para una muestra transparente a la luz y que representa el ruido de fondo. Se puede apreciar un aumento de la señal OA a los $15.3 \mu$ s y cuya duración es de $0.9 \mu \mathrm{s}$. A partir de estos datos y el valor de la velocidad del sonido, se puede obtener la distancia entre la superficie de la muestra y el sensor $(22.8 \mathrm{~mm})$ y el diámetro del fantoma $(1.3 \mathrm{~mm})$. Los valores encontrados concuerdan con el diámetro de la esfera de colorante y con la distancia focal del sensor piezoeléctrico.

\section{CONCLUSIONES}

En este trabajo se implementó un sistema de iluminación basado en LEDs y en el sistema embebido EDU-CIAA. Éste puede emitir pulsos con una elevada frecuencia $(>1$ $\mathrm{kHz}$ ), con una duración menor al microsegundo y con intensidades suficientemente elevadas para detectar señales OA. Se destaca que el uso combinado de LEDs y EDUCIAA tiene la posibilidad de integrar y ampliar nuevas funcionalidades, tales como esquemas de codificación para mejorar la relación señal a ruido [10] y arreglos de LEDs de distintas longitudes de onda para implementar sistemas multi-espectrales [31].

A partir de los resultados encontrados en este trabajo y con el objetivo de obtener mayores energías de emisión óptica, se planea la realización de un nuevo prototipo con varias mejoras en el layout, en las cuales se destacan el reemplazo del driver por otro capaz de entregar mayor corriente máxima, y la modificación del montaje del LED.

Finalmente, a modo de comparación, sistemas comerciales como el Cyberdyne Acoustic X [32] tienen un rango más acotado de frecuencias que suele estar entre $1 \mathrm{kHz}$ y $4 \mathrm{kHz}$. Por otro lado, si bien el sistema comercial puede entregar pulsos de menor duración ( $30 \mathrm{~ns}$ ), ambos poseen una resolución de 5 ns. Otro punto a destacar es que el sistema Acoustic X utiliza arreglos de LEDs de distintas longitudes, característica que puede reproducirse en el sistema propuesto gracias a la disponibilidad de más canales PWM en el sistema embebido EDU-CIAA. 


\section{AGRADECIMIENTOS}

Este trabajo fue apoyado por los subsidios de la ANPCyT (PICT 2016-2204 y PICT 2018-04589) y de la Universidad de Buenos Aires (UBACyT 20020190100032BA).

\section{REFERENCIAS}

[1] M. Xu and L. Wang, "Photoacoustic imaging in biomedicine," Rev. Sci. Instrum., vol. 77, pp. 041 101-1-23, 2006.

[2] L. V. Wang, "Tutorial on photoacoustic microscopy and computed tomography," IEEE J. of Selected Topics in Quantum Electronics, vol. 14, pp. 171-179, 2008.

[3] J. Bauer-Marschallinger, K. Felbermayer, and T. Berer, "All-optical photoacoustic projection imaging," J. Biomed. Opt. Express, vol. 8, pp. 3938-3951, 2017.

[4] G. Paltauf, P. Hartmair, G. Kovachev, and R. Nuster, "Piezoelectric line detector array for photoacoustic tomography," Photoacoustics, vol. 8, pp. 28-36, 2017.

[5] A. Hariri, J. Lemaster, J. Wang, A. Jeevarathinam, D. Chao, and J. Jokerst, "The characterization of an economic and portable LEDbased photoacoustic imaging system to facilitate molecular imaging," Photoacoustics, vol. 9, pp. 10-20, 2018.

[6] P. Upputuri and M. Pramanik, "Fast photoacoustic imaging systems using pulsed laser diodes: a review," Biomed. Eng. Lett., vol. 2018, pp. 167-181, 2018.

[7] R. Ma, A. Taruttis, V. Ntziachristos, and D. Razansky, "Multispectral optoacoustic tomography (msot) scanner for whole-body small animal imaging," Opt. Express, vol. 17, pp. 21414-1-13, 2009.

[8] S. Agrawal, C. Fadden, A. Dangi, X. Yang, H. Albahrani, N. Frings, S. Zadi, and S. Kothapalli, "Light-emitting-diode-based multispectral photoacoustic computed tomography system," Sensors, vol. 19, pp. 4861-1-13, 2019.

[9] H. Zhong, T. Duan, H. Lan, M. Zhou, and F. Fao, "Review of lowcost photoacoustic sensing and imaging based on laser diode and light-emitting diode," Sensors, vol. 18, pp. 1-24, 2018.

[10] T. Allen and P. Beard, "High power visible light emitting diodes as pulsed excitation sources for biomedical photoacoustics," Biomed. Opt. Express, vol. 7, pp. 1261-1270, 2016.

[11] M. Singh, LED-Based Photoacoustic Imaging. Springer, 2020.

[12] "Proyecto computadora industrial abierta argentina," Argentina. [Online]. Available: http://www.proyecto-ciaa.com.ar/

[13] M. G. Gonzalez, B. Abadi, L. C. Brazzano, and P. Sorichetti, "Linear piezoelectric sensor for optoacoustic tomography: electroacoustic characterization," in Proc. IEEE Argencon, 2018, pp. 1-4.

[14] M. G. Gonzalez, P. Sorichetti, and G. Santiago, "Reducing the capacitance of piezoelectric film sensors," Rev. Sci. Instrum., vol. 87 pp. 045 003-1-5, 2016.

[15] R. Insabella, M. Gonzalez, E. Acosta, and G. Santiago, "Dielectric antenna effects in integrating line piezoelectric sensors for optoacoustic imaging," Meas. Sci. and Tech., vol. 31, p. 125103, 2020.

[16] R. Insabella, M. Gonzalez, L. Riobo, K. Hass, and F. Veiras, "Software-defined optoacoustic tomography," Appl. Opt., vol. 59, pp. 706-711, 2020.

[17] "LED-CBT-120-G-C11 datasheet," Luminus. [Online]. Available: https://download.luminus.com/datasheets/Luminus

[18] "MOSFET-IRF8707 datasheet," International Rectifier. [Online]. Available: https://www.infineon.com/dgdl/irf8707pbf.pdf?fileId= $5546 \mathrm{~d} 462533600 \mathrm{a} 40153560 \mathrm{~d} 57 \mathrm{f} 81 \mathrm{~d} 6 \mathrm{~b}$

[19] "MOSFET driver TC4427 datasheet," Microchip Technology. [Online]. Available: https://www.microchip.com/wwwproducts/en/TC4427

[20] M. G. Gonzalez, L. Riobo, L. C. Brazzano, F. Veiras, P. Sorichetti, and G. Santiago, "Generation of sub-microsecond quasi-unipolar pressure pulses," Ultrasonics, vol. 98, pp. 15-19, 2019.

[21] M. G. Gonzalez, E. Acosta, and G. Santiago, "Simple method to determine the resolution and sensitivity of systems for optoacoustic tomography," Revista Elektron, vol. 2, pp. 63-66, 2018.

[22] P. Ninni, F. Martelli, and G. Zaccanti, "The use of india ink in tissuesimulating phantoms," Opt. Express, vol. 18, no. 26, pp. 26854-1-12, 2010.

[23] "Laser Energy Sensor datasheet," Coherent. [Online]. Available: https://www.coherent.com/measurementcontrol/measurement/multi purpose-energymax-sensors

[24] "Optical receiver 818-BB-21 datasheet," Newport. [Online]. Available: https://www.newport.com/p/818-BB-21A

[25] "High Speed Current Amplifier datasheet," FEMTO. [Online]. Available: https://www.femto.de/images/pdf-dokumente/de-hca-100m-50kc.pdf
[26] K. Mackenzie, "Nine-term equation for sound speed in the oceans," J. Acousti. Soc. Am., vol. 70, pp. 807-812, 1981.

[27] P. Binh, V. Trong, P. Renucci, and X. Marie, "A simple subnanosecond ultraviolet light pulse generator with high repetition rate and peak power," Rev. Sci. Instrum., vol. 84, pp. 083 102-1-7, 2013.

[28] "LTspice," Analog Devices. [Online]. Available: https://www.analog.com/en/design-center/design-tools-andcalculators/ltspice-simulator.html

[29] T. Kishi, H. Tanaka, Y. Umeda, and O. Takyu, "A high-speed LED driver that sweeps out the remaining carriers for visible light communications," J. Lightw. Technol., vol. 32, pp. 239-248, 2014.

[30] H. Halbritter, C. Jaeger, R. Weber, M. Schwind, and F. Moellmer, "High-speed LED driver for ns-pulse switching of high-current leds," IEEE Photonics Tech. Lett., vol. 26, pp. 1871-1873, 2014.

[31] H. Zhang, K. Kondo, M. Yamakawa, and T. Shiina, "Simultaneous multispectral coded excitation using periodic and unipolar msequences for photoacoustic imaging," in Proc. SPIE, vol. 8581, no. 85812Y, 2013.

[32] "Cyberdyne Acoustic X," Japan. [Online]. Available: https://www.cyberdyne.jp/english/products/pdf/AcousticX-flyerA4Size-English.pdf 